\title{
Status of Chiral-Scale Perturbation Theory
}

\author{
R. J. Crewther \\ CSSM and ARC Centre of Excellence for Particle Physics at the Tera-scale, \\ Department of Physics, University of Adelaide, \\ Adelaide, South Australia 5005, Australia \\ E-mail: rcrewthe@physics.adelaide.edu.au
}

\section{Lewis C. Tunstall*}

Albert Einstein Center for Fundamental Physics, Institute for Theoretical Physics, University of Bern, Sidlerstrasse 5, CH-3012 Bern, Switzerland

E-mail: tunstalleitp.unibe.ch

\begin{abstract}
Chiral-scale perturbation theory $\chi \mathrm{PT}_{\sigma}$ has been proposed as an alternative to chiral $S U(3)_{L} \times$ $S U(3)_{R}$ perturbation theory which explains the $\Delta I=1 / 2$ rule for kaon decays. It is based on a lowenergy expansion about an infrared fixed point in three-flavor QCD. In $\chi \mathrm{PT}_{\sigma}$, quark condensation $\langle\bar{q} q\rangle_{\text {vac }} \neq 0$ induces nine Nambu-Goldstone bosons: $\pi, K, \eta$ and a QCD dilaton $\sigma$ which we identify with the $f_{0}(500)$ resonance. Partial conservation of the dilatation and chiral currents constrains low-energy constants which enter the effective Lagrangian of $\chi \mathrm{PT}_{\sigma}$. These constraints allow us to obtain new phenomenological bounds on the dilaton decay constant via the coupling of $\sigma / f_{0}$ to pions, whose value is known precisely from dispersive analyses of $\pi \pi$ scattering. Improved predictions for $\sigma \rightarrow \gamma \gamma$ and the $\sigma N N$ coupling are also noted. To test $\chi \mathrm{PT}_{\sigma}$ for kaon decays, we revive a 1985 proposal for lattice methods to be applied to $K \rightarrow \pi$ on-shell.
\end{abstract}

The 8th International Workshop on Chiral Dynamics, CD2015

29 June 2015 - 03 July 2015

Pisa, Italy

\footnotetext{
*Speaker.
} 


\section{Approximate Scale Invariance in Low-Energy QCD}

In the low-energy regime of QCD with heavy quarks $t, b, c$ decoupled, the relevance of scale (dilatation) invariance is determined by the trace anomaly [1]-[4] of the resulting 3-flavor theory: ${ }^{1}$

$$
\theta_{\mu}^{\mu}=\frac{\beta\left(\alpha_{s}\right)}{4 \alpha_{s}} G_{\mu \nu}^{a} G^{a \mu v}+\left(1+\gamma_{m}\left(\alpha_{s}\right)\right) \sum_{q=u, d, s} m_{q} \bar{q} q
$$

Depending on the infrared behaviour of $\beta$, there are only two realistic scenarios (Fig. 1 (A)):

1. If $\beta$ remains negative and non-zero, possibly diverging linearly at large $\alpha_{s}$, scale invariance is explicitly broken by $\theta_{\mu}^{\mu}$ being large as an operator. There is no hint of approximate scale invariance: quantities such as the nucleon mass $M_{N}=\left\langle N\left|\theta_{\mu}^{\mu}\right| N\right\rangle$ are generated almost entirely by the gluonic term in (1.1). Then conventional chiral $S U(3)_{L} \times S U(3)_{R}$ perturbation theory $\chi \mathrm{PT}_{3}$ is the appropriate low-energy effective theory for QCD amplitudes expanded in powers of $O\left(m_{K}\right)$ external momenta and light quark masses $m_{u, d, s}=O\left(m_{K}^{2}\right)$.

2. If $\beta$ vanishes when $\alpha_{s}$ runs non-perturbatively to an infrared fixed point $\alpha_{\mathrm{IR}}$, the gluonic term $\sim G_{\mu \nu}^{a} G^{a \mu v}$ in (1.1) is absent and the dilatation current $D_{\mu}=x^{v} \theta_{\mu \nu}$ becomes conserved in the limit of vanishing quark masses:

$$
\begin{aligned}
\left.\partial^{\mu} D_{\mu}\right|_{\alpha_{s}=\alpha_{\mathrm{IR}}}=\left.\theta_{\mu}^{\mu}\right|_{\alpha_{s}=\alpha_{\mathrm{IR}}} & =\left(1+\gamma_{m}\left(\alpha_{\mathrm{IR}}\right)\right) \sum_{q=u, d, s} m_{q} \bar{q} q \\
& \rightarrow 0, S U(3)_{L} \times S U(3)_{R} \text { limit. }
\end{aligned}
$$

Although the Hamiltonian preserves dilatations in this limit, the vacuum state is not scale invariant due to the formation of a quark condensate $\langle\bar{q} q\rangle_{\text {vac }} \neq 0$. As a result, both chiral $S U(3)_{L} \times S U(3)_{R}$ and scale symmetry are realized in the Nambu-Goldstone (NG) mode and the spectrum contains nine massless bosons: $\pi, K, \eta$ and a $0^{++}$QCD dilaton $\sigma$. Non-NG bosons remain massive despite the vanishing of $\theta_{\mu}^{\mu}$ and have their scale set by $\langle\bar{q} q\rangle_{\mathrm{vac}}$. The relevant low-energy expansion involves a combined limit

$$
m_{u, d, s} \sim 0 \quad \text { and } \quad \alpha_{s} \lesssim \alpha_{\mathrm{IR}}
$$

and leads to a new effective theory $\chi \mathrm{PT}_{\sigma}$ of approximate chiral-scale symmetry $[5,6] . \mathrm{In}$ this scenario, the dilaton mass is set by $m_{s}$, so the natural candidate for $\sigma$ is the $f_{0}(500)$ resonance, a broad $0^{++}$state whose complex pole mass has real part $\lesssim m_{K}[7,8,9]$.

Until now, scenario 1 has been the generally accepted view, but we have observed $[5,6]$ that $\chi \mathrm{PT}_{\sigma}$ offers several advantages over $\chi \mathrm{PT}_{3}$ : it explains the mass and width of $f_{0}(500)$, produces convergent chiral expansions as a result of $\sigma / f_{0}$ being promoted to the NG sector, and most importantly, explains the $\Delta I=1 / 2$ rule for non-leptonic $K$ decays (Fig. 1 (B)).

Because approximate scale symmetry is included, the effective Lagrangian for $\chi \mathrm{PT}_{\sigma}$ (Sec. 2) contains several new low-energy constants (LECs) yet to be determined precisely from data. Of particular interest is the dilaton decay constant $F_{\sigma}$ given by $m_{\sigma}^{2} F_{\sigma}=-\left\langle\sigma\left|\theta_{\mu}^{\mu}\right| \mathrm{vac}\right\rangle$. If $F_{\sigma}$ is roughly 100

\footnotetext{
${ }^{1}$ Here, $G_{\mu \nu}^{a}$ is the gluon field strength, $\alpha_{s}=g_{s}^{2} / 4 \pi$ is the strong running coupling, and $\beta=\mu^{2} \partial \alpha_{s} / \partial \mu^{2}$ and $\gamma_{m}=\mu^{2} \partial \ln m_{q} / \partial \mu^{2}$ refer to a mass-independent renormalization scheme with scale $\mu$.
} 


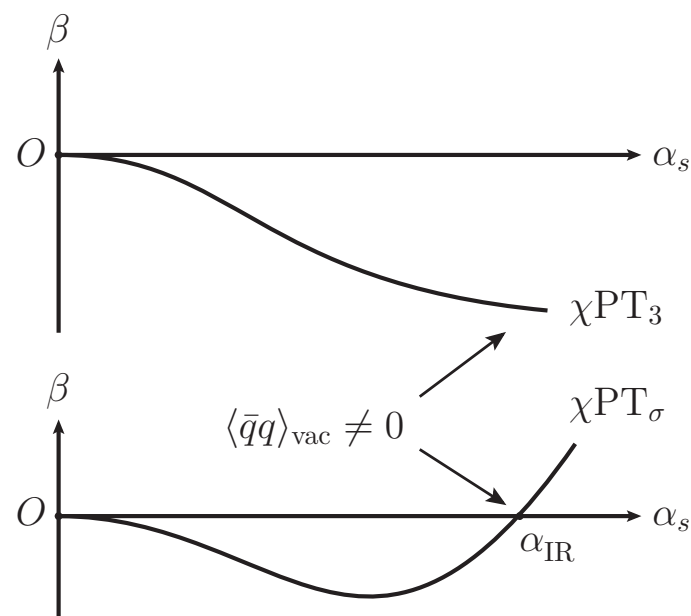

$(\mathrm{A})$
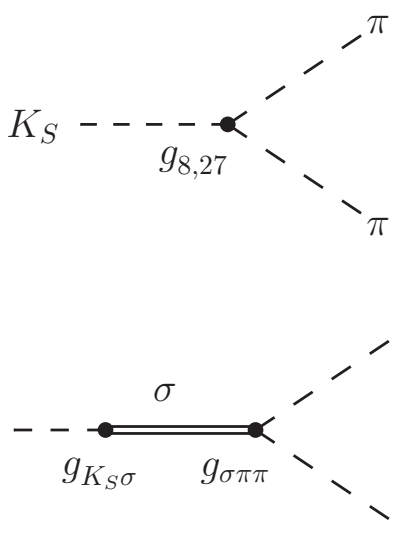

(B)

Figure 1: (A) Scenarios for the $\beta$ function in three-flavor QCD, with corresponding low-energy expansions. In the absence of an infrared fixed point $\alpha_{\mathrm{IR}}$ (top diagram), there is no approximate scale invariance and chiral $S U(3)_{L} \times S U(3)_{R}$ perturbation theory $\chi \mathrm{PT}_{3}$ is relevant at low-energies. If $\alpha_{\mathrm{IR}}$ exists (bottom diagram), quark condensation $\langle\bar{q} q\rangle_{\text {vac }} \neq 0$ implies that the NG spectrum contains a QCD dilaton $\sigma$, and $\chi \mathrm{PT}_{3}$ must be replaced by chiral-scale perturbation theory $\chi \mathrm{PT}_{\sigma}$. (B) Diagrams for $K \rightarrow \pi \pi$ decay in lowest-order $\chi \mathrm{PT}_{\sigma}$. The dilaton pole diagram is responsible for the dominant $\Delta I=1 / 2$ amplitude.

$\mathrm{MeV}$, scale breaking by the vacuum can generate large masses such as $m_{N} \approx F_{\sigma} g_{\sigma N N}$ (GoldbergerTreiman relation for dilatons [10]) for $m_{\sigma}$ small. The imprecise value of $F_{\sigma}$ in our previous work $[5,6]$ arose from large uncertainties in the phenomenological value of $g_{\sigma N N}[11,12]$.

We circumvent this difficulty in Secs. 3 and 4. First, we find new constraints on LECs in the $\chi \mathrm{PT}_{\sigma}$ effective Lagrangian by requiring full consistency with the dilatation and chiral currents being conserved in the limit (1.2). These constraints allow us to determine $F_{\sigma}$ from the $\sigma \pi \pi$ coupling, whose value is known to remarkable precision from dispersive analyses $[7,8,9]$ of $\pi \pi$ scattering. Then we obtain improved predictions for the non-perturbative Drell-Yan ratio

$$
R=\sigma\left(e^{+} e^{-} \rightarrow \text { hadrons }\right) / \sigma\left(e^{+} e^{-} \rightarrow \mu^{+} \mu^{-}\right) \quad \text { at } \alpha_{\mathrm{IR}},
$$

as well as the $\sigma N N$ coupling.

In Sec. 5, we resurrect an old proposal [13] to apply lattice QCD for $K \rightarrow \pi$ on-shell to determine the couplings $g_{8,27}$ in Fig. 1 (B). Comments on the validity of $\chi \mathrm{PT}_{\sigma}$ are reviewed in Sec. 6 .

\section{Chiral-Scale Lagrangian}

For strong interactions, the most general effective Lagrangian of $\chi \mathrm{PT}_{\sigma}$ is of the form

$$
\mathscr{L}_{\chi \mathrm{PT}_{\sigma}}=: \mathscr{L}_{\text {inv }}^{d=4}+\mathscr{L}_{\text {anom }}^{d>4}+\mathscr{L}_{\text {mass }}^{d<4}:,
$$

where

$$
d_{\mathrm{anom}}=4+\gamma_{G^{2}}\left(\alpha_{s}\right) \quad \text { and } \quad d_{\text {mass }}=3-\gamma_{m}\left(\alpha_{s}\right)
$$


are the respective scaling dimensions of $G_{\mu \nu}^{a} G^{a \mu v}$ and $\bar{q} q$. In lowest order (LO) of the chiral-scale expansion, we have $\gamma_{m}=\gamma_{m}\left(\alpha_{\mathrm{IR}}\right)$ and

$$
\gamma_{G^{2}}\left(\alpha_{s}\right) \equiv \beta^{\prime}\left(\alpha_{s}\right)-\beta\left(\alpha_{s}\right) / \alpha_{s}=\beta^{\prime}\left(\alpha_{\mathrm{IR}}\right)+O\left(\alpha_{s}-\alpha_{\mathrm{IR}}\right),
$$

so the resulting terms in (2.1) are

$$
\begin{aligned}
\mathscr{L}_{\text {inv }, \mathrm{LO}}^{d=4} & =\left\{c_{1} \mathscr{K}+c_{2} \mathscr{K}_{\sigma}+c_{3} e^{2 \sigma / F_{\sigma}}\right\} e^{2 \sigma / F_{\sigma}}, \\
\mathscr{L}_{\text {anom }, \mathrm{LO}}^{d>4} & =\left\{\left(1-c_{1}\right) \mathscr{K}+\left(1-c_{2}\right) \mathscr{K}_{\sigma}+c_{4} e^{2 \sigma / F_{\sigma}}\right\} e^{\left(2+\beta^{\prime}\right) \sigma / F_{\sigma}}, \\
\mathscr{L}_{\text {mass }, \mathrm{LO}}^{d<4} & =\operatorname{Tr}\left(M U^{\dagger}+U M^{\dagger}\right) e^{\left(3-\gamma_{m}\right) \sigma / F_{\sigma}},
\end{aligned}
$$

where

$$
\mathscr{K}=\frac{1}{4} F_{\pi}^{2} \operatorname{Tr}\left(\partial_{\mu} U \partial^{\mu} U^{\dagger}\right) \quad \text { and } \quad \mathscr{K}_{\sigma}=\frac{1}{2}\left(\partial_{\mu} \sigma\right)^{2} .
$$

As $\alpha_{s} \rightarrow \alpha_{\mathrm{IR}}$, the gluonic anomaly vanishes, so $\mathscr{L}_{\text {anom }}=O\left(\partial^{2}, M\right)$ and we must set $c_{4}=O(M)$. Vacuum stability in the $\sigma$ direction about $\sigma=0$ (no tadpoles) implies

$$
\begin{aligned}
4 c_{3}+\left(4+\beta^{\prime}\right) c_{4} & =-\left(3-\gamma_{m}\right)\left\langle\operatorname{Tr}\left(M U^{\dagger}+U M^{\dagger}\right)\right\rangle_{\mathrm{vac}} \\
& =-\left(3-\gamma_{m}\right) F_{\pi}^{2}\left(m_{K}^{2}+\frac{1}{2} m_{\pi}^{2}\right),
\end{aligned}
$$

so $c_{3}$ is also $O(M)$. Expanding (2.4) about $\sigma=0$ and $U=I$ yields the $\sigma \pi \pi$ coupling

$$
\mathscr{L}_{\sigma \pi \pi}=\left\{\left[2+\left(1-c_{1}\right) \beta^{\prime}\right]|\partial \pi|^{2}-\left(3-\gamma_{m}\right) m_{\pi}^{2}|\pi|^{2}\right\} \sigma /\left(2 F_{\sigma}\right),
$$

while the corresponding $\sigma \pi \pi$ vertex for an on-shell dilaton is

$$
g_{\sigma \pi \pi}=-\frac{1}{2 F_{\sigma}}\left\{\left[2+\left(1-c_{1}\right) \beta^{\prime}\right] m_{\sigma}^{2}+2\left[1-\gamma_{m}-\left(1-c_{1}\right) \beta^{\prime}\right] m_{\pi}^{2}\right\} .
$$

\section{Effective Energy-Momentum Tensor and its Trace}

In any field theory, the energy-momentum tensor can be identified by adding a gravitational source field $g_{\mu v}(x)$ coupled to matter fields in a generally covariant fashion. In $\chi \mathrm{PT}_{\sigma}$, this amounts to the substitution

$$
\mathscr{L}_{\chi \mathrm{PT}_{\sigma}}\left[U, U^{\dagger}, \sigma\right] \rightarrow \mathscr{L}_{\chi \mathrm{PT}_{\sigma}}\left[U, U^{\dagger}, \sigma, g_{\mu \nu}\right],
$$

where the new effective Lagrangian must be constructed in terms of generally covariant operators. Then the energy-momentum tensor is defined via the variation

$$
\theta_{\mu v}(x)=2\left[\frac{\delta}{\delta g^{\mu v}(x)} \sqrt{-g} \mathscr{L}\left[U, U^{\dagger}, \sigma, g_{\mu v}\right]\right]_{g_{\mu \nu}=\eta_{\mu \nu}},
$$

where $g=\operatorname{det}\left(g_{\mu \nu}\right)$ is the determinant of the metric tensor and $\eta_{\mu \nu}$ is the flat Minkowski metric. Generalising Donoghue and Leutwyler [14], we obtain the lowest order result

$$
\begin{aligned}
\theta_{\mu \nu}= & {\left[\frac{1}{2} F_{\pi}^{2} \operatorname{Tr}\left(\partial_{\mu} U \partial_{\nu} U^{\dagger}\right)-g_{\mu \nu} \mathscr{K}\right]\left[c_{1} e^{2 \sigma / F_{\sigma}}+\left(1-c_{1}\right) e^{\left(2+\beta^{\prime}\right) \sigma / F_{\sigma}}\right] } \\
& +\left(\partial_{\mu} \sigma \partial_{\nu} \sigma-g_{\mu \nu} \mathscr{K}_{\sigma}\right)\left[c_{2} e^{2 \sigma / F_{\sigma}}+\left(1-c_{2}\right) e^{\left(2+\beta^{\prime}\right) \sigma / F_{\sigma}}\right] \\
& -g_{\mu \nu} \operatorname{Tr}\left(M U^{\dagger}+U M^{\dagger}\right) e^{\left(3-\gamma_{m}\right) \sigma / F_{\sigma}}-g_{\mu \nu} e^{4 \sigma / F_{\sigma}}\left(c_{3}+c_{4} e^{\beta^{\prime} \sigma / F_{\sigma}}\right) .
\end{aligned}
$$


The trace of (3.3) involves scale invariant operators like $\operatorname{Tr}\left(\partial_{\mu} U \partial^{\mu} U^{\dagger}\right) e^{2 \sigma / F_{\sigma}}$ which obscure the connection between the scale invariance and a conserved dilatation current $D_{\mu}$. To remedy this, we "improve" $\theta_{\mu \nu}[15]$ by adding a term

$$
I_{\mu \nu}=\frac{F_{\sigma}^{2}}{6}\left(g_{\mu \nu} \partial^{2}-\partial_{\mu} \partial_{v}\right)\left[c_{2} e^{2 \sigma / F_{\sigma}}+\frac{2\left(1-c_{2}\right)}{2+\beta^{\prime}} e^{\left(2+\beta^{\prime}\right) \sigma / F_{\sigma}}\right]
$$

such that the trace of

$$
\left.\theta_{\mu v}\right|_{\mathrm{eff}}=\theta_{\mu v}+I_{\mu v}
$$

is given entirely in terms of explicit scale-breaking operators $\mathscr{L}_{d}$ of scale dimension $d$ :

$$
\left.\partial^{\mu} D_{\mu}\right|_{\mathrm{eff}}=\left.\theta_{\mu}^{\mu}\right|_{\mathrm{eff}}=\sum_{d}(d-4) \mathscr{L}_{d}
$$

Explicitly, the improved trace is

$$
\begin{aligned}
\left.\theta_{\mu}^{\mu}\right|_{\mathrm{eff}}= & \beta^{\prime} \mathscr{L}_{\text {anom }}^{d>4}-\left(1+\gamma_{m}\right) \mathscr{L}_{\text {mass }}^{d<4} \\
= & \beta^{\prime}\left\{\left(1-c_{1}\right) \mathscr{K}+\left(1-c_{2}\right) \mathscr{K}_{\sigma}+c_{4} e^{2 \sigma / F_{\sigma}}\right\} e^{\left(2+\beta^{\prime}\right) \sigma / F_{\sigma}} \\
& -\left(1+\gamma_{m}\right) \operatorname{Tr}\left(M U^{\dagger}+U M^{\dagger}\right) e^{\left(3-\gamma_{m}\right) \sigma / F_{\sigma}}
\end{aligned}
$$

It vanishes in the chiral-scale limit (1.2) only if the low-energy constants associated with $d>4$ operators satisfy

$$
c_{1}=c_{2}=1, \quad \text { for } m_{u, d, s} \rightarrow 0 \text { and } \alpha_{s} \rightarrow \alpha_{\mathrm{IR}},
$$

in addition to the condition $c_{4}=O(M)$ required by tadpole cancellation (2.6). Note that the condition $c_{1} \rightarrow 1$ in (3.8) ensures that chiral currents have vanishing anomalous dimensions. We can summarise these LO conditions by writing

$$
c_{i}=1+O(M), \quad i=1,2,
$$

where the $O(M)$ term is a linear superposition of $O\left(p^{2}, M\right)$ operators and associated LECs.

\section{Improved Predictions}

An immediate consequence of the constraint (3.9) is that the $\sigma \pi \pi$ coupling for an on-shell dilaton (2.8) takes a particularly simple form

$$
g_{\sigma \pi \pi}=-\frac{1}{F_{\sigma}}\left[m_{\sigma}^{2}+\left(1-\gamma_{m}\right) m_{\pi}^{2}\right], \quad \text { where }-1 \leq 1-\gamma_{m}<2 .
$$

Since the narrow-width approximation is valid in lowest order $\chi \mathrm{PT}_{\sigma}[6]$, we have

$$
\Gamma_{\sigma \pi \pi}=\frac{\left|g_{\sigma \pi \pi}\right|^{2}}{16 \pi m_{\sigma}} \sqrt{1-4 m_{\pi}^{2} / m_{\sigma}^{2}},
$$

and this allows us to obtain bounds on $F_{\sigma}$ from dispersive analyses of $\pi \pi$ scattering based on the Roy equations. For example, the $f_{0} / \sigma$ 's mass and width from [7]

$$
m_{\sigma}=441_{-8}^{+16} \mathrm{MeV}, \quad \Gamma_{\sigma \pi \pi}=544_{-25}^{+18} \mathrm{MeV},
$$


constrain $F_{\sigma}$ to lie within the interval $44 \mathrm{MeV} \leq F_{\sigma} \leq 61 \mathrm{MeV}$, where we have allowed $1-\gamma_{m}$ to vary according to (4.1). For the moment, we assume that NLO corrections are not a problem.

With $F_{\sigma}$ fixed in this manner, we can now use the Golberger-Treiman relation for dilatons [10] to predict the value for the $\sigma N N$ coupling. We find $16 \leq g_{\sigma N N} \leq 21$, which is somewhat larger than previous phenomenological determinations $[11,12]$. Another important application concerns $\sigma \rightarrow \gamma \gamma$, where an analysis $[5,6]$ of the electromagnetic trace anomaly in $\chi \mathrm{PT}_{\sigma}$ relates the $\sigma \gamma \gamma$ coupling to (1.4):

$$
g_{\sigma \gamma \gamma}=\frac{2 \alpha}{3 \pi F_{\sigma}}\left(R_{\mathrm{IR}}-\frac{1}{2}\right) \text {. }
$$

By fixing $g_{\sigma \gamma \gamma}$ from the di-photon width $\Gamma_{\sigma \gamma \gamma}=2.0 \pm 0.2 \mathrm{keV}$ [16], we find $2.4 \leq R_{\mathrm{IR}} \leq 3.1$, which is to be compared with our previous estimate $R_{\mathrm{IR}} \approx 5[5,6]$.

\section{Proposal to test $K \rightarrow \pi$ on the Lattice}

The key idea [13] is to keep both $K$ and $\pi$ on shell and allow $O\left(m_{K}\right)$ momentum transfers.

The lowest-order diagrams for the decay $K \rightarrow \pi \pi$ in Fig. 1 (B) are derived from an effective weak $\chi \mathrm{PT}_{\sigma}$ Lagrangian $[5,6]$

$$
\mathscr{L}_{\text {weak }}=Q_{8} \sum_{n} g_{8 n} e^{\left(2-\gamma_{8 n}\right) \sigma / F_{\sigma}}+g_{27} Q_{27} e^{\left(2-\gamma_{27}\right) \sigma / F_{\sigma}}+Q_{m w} e^{\left(3-\gamma_{m w}\right) \sigma / F_{\sigma}}+\text { h.c. }
$$

which reduces to the standard $\chi \mathrm{PT}_{3}$ Lagrangian

$$
\left.\mathscr{L}_{\text {weak }}\right|_{\sigma=0}=g_{8} Q_{8}+g_{27} Q_{27}+Q_{m w}+\text { h.c. }
$$

in the limit $\sigma \rightarrow 0$. Eqs. (5.1) and (5.2) contain an octet operator [17]

$$
Q_{8}=J_{13}^{\mu} J_{\mu 21}-J_{23}^{\mu} J_{\mu 11}, \quad J_{i j}^{\mu}=\left(U \partial^{\mu} U^{\dagger}\right)_{i j}
$$

the $U$-spin triplet component $[13,18]$ of a $\mathbf{2 7}$ operator

$$
Q_{27}=J_{13}^{\mu} J_{\mu 21}+\frac{3}{2} J_{23}^{\mu} J_{\mu 11}
$$

and a weak mass operator [19]

$$
Q_{m w}=\operatorname{Tr}\left(\lambda_{6}-i \lambda_{7}\right)\left(g_{M} M U^{\dagger}+\bar{g}_{M} U M^{\dagger}\right) .
$$

Powers of $e^{\sigma / F_{\sigma}}$ are used to adjust the operator dimensions of $Q_{8}, Q_{27}$, and $Q_{m w}$ in (5.1), with octet quark-gluon operators allowed to have differing dimensions at $\alpha_{\mathrm{IR}}$.

In 1985, it was observed [13] that the isospin- $\frac{1}{2}$ term $Q_{m w}$ in Eq. (5.2), when combined with the strong mass term, would be removed by vacuum realignment and therefore could not help solve the $\Delta I=1 / 2$ puzzle. In $\chi \mathrm{PT}_{\sigma}$, the outcome is different $[5,6]$ due to the $\sigma$ dependence of the $Q_{m w}$ term in Eq. (5.1). Provided there is a mismatch between the weak mass operator's dimension $\left(3-\gamma_{m w}\right)$ and the dimension $\left(3-\gamma_{m}\right)$ of $\mathscr{L}_{\text {mass }}$, the $\sigma$ dependence of $Q_{m w} e^{\left(3-\gamma_{m w}\right) / F_{\sigma}}$ cannot be eliminated by a chiral rotation. As a result, there is a residual interaction $\mathscr{L}_{K_{S} \sigma}=g_{K_{S} \sigma} K_{S} \sigma$ which mixes $K_{S}$ and $\sigma$ in lowest $O\left(p^{2}\right)$ order $^{2}$

$$
g_{K_{S} \sigma}=\left(\gamma_{m}-\gamma_{m w}\right) \operatorname{Re}\left\{\left(2 m_{K}^{2}-m_{\pi}^{2}\right) \bar{g}_{M}-m_{\pi}^{2} g_{M}\right\} F_{\pi} / F_{\sigma}
$$

\footnotetext{
${ }^{2}$ We have corrected a factor of 2 in the formula for the $K_{S} \sigma$ coupling in our original papers [5, 6].
} 
and produces the $\Delta I=1 / 2 \sigma$-pole amplitude of Fig. 1 (B).

The $\chi \mathrm{PT}_{3}$ analysis of 1985 [13] included a suggestion that kaon decays be tested by applying lattice QCD to the weak process $K \rightarrow \pi$, with both $K$ and $\pi$ on shell. It was made at a time when low-lying scalar resonances $\left(\varepsilon(700)\right.$ before $1974, f_{0}(500)$ since 1996$)$ were thought not to exist.

This proposal now needs to be taken seriously because:

- Lattice calculations are much easier with only two particles on shell instead of the three in $K \rightarrow \pi \pi$ (all on shell) being analysed by the RBC/UKQCD collaborations [20, 21].

- The 1985 analysis is easily extended to $\chi \mathrm{PT}_{\sigma}$ by including $\sigma / f_{0}$ pole amplitudes in chiral Ward identities connecting on-shell $K \rightarrow \pi \pi$ to $K \rightarrow \pi$ on shell. The no-tadpoles theorem

$$
\left\langle K\left|\mathscr{H}_{\text {weak }}\right| \text { vac }\right\rangle=O\left(m_{s}^{2}-m_{d}^{2}\right), K \text { on shell, }
$$

remains valid.

- The lattice result for $K \rightarrow \pi \pi$ on-shell will not distinguish $\Delta I=1 / 2$ contributions from the $g_{8}$ contact diagram and the $\sigma / f_{0}$ pole diagram in Fig. 1 (B). A lattice calculation of $K \rightarrow \pi$ on shell would measure $g_{8}$ (and $g_{27}$ ) directly, with no interference from $\sigma / f_{0}$ poles. Then we would finally learn whether $g_{8}$ is unnaturally large or not.

A key feature of the proposal is that the operator in the on-shell amplitude $\left\langle\pi\left|\left[F_{5}, \mathscr{H}_{\text {weak }}\right]\right| K\right\rangle$ necessarily carries non-zero momentum $q^{\mu}=O\left(m_{K}\right)$. For either $\chi \mathrm{PT}_{\sigma}$ or $\chi \mathrm{PT}_{3}$, the $K \rightarrow \pi$ amplitude can be evaluated in the range

$$
-m_{K}^{2} \lesssim q^{2} \leqslant\left(m_{K}-m_{\pi}\right)^{2}
$$

We highlight the point $q^{\mu} \neq 0$ because since 1985, there has been a widespread misconception in the literature ${ }^{3}$ that the analysis [13] involved setting $q^{\mu}=0$ as in [19], with the pion in $K \rightarrow \pi$ sent off shell via an interpolating operator. There was and is no reason for this. For example, when writing a soft meson theorem for $\Sigma \rightarrow p \pi$, it is not necessary to force one of the baryons off shell.

\section{Issues}

When considering the validity of $\chi \mathrm{PT}_{\sigma}$, it is important to avoid any presumption that dimensional transmutation necessarily implies that $\theta_{\mu}^{\mu}$ is large and $\neq 0$. Implicit in this intuition is a prejudice that scale invariance cannot be strongly broken via the vacuum when $\theta_{\mu}^{\mu} \rightarrow 0$. If the dilaton is a true NG boson, i.e. $m_{\sigma} \rightarrow 0$ with $F_{\sigma} \neq 0$ for $\theta_{\mu}^{\mu} \rightarrow 0$, it can couple to mass insertion terms in Callan-Symanzik equations and cause them to be non-zero in the zero-mass limit. Then Green's functions do not exhibit the power-law scaling expected for manifestly scale-invariant field theories.

This point is illustrated for the quark condensate in Fig. 1 (A). In scenario 1 (top diagram), the running of $\alpha_{s}$ is driven by the presence of quantities like $\langle\bar{q} q\rangle_{\text {vac }}$ (a mechanism often cited in papers on walking gauge theories [22]). In scenario 2 (bottom diagram), the running coupling freezes at $\alpha_{\mathrm{IR}}$, where the condensate is a scale-breaking property of the vacuum.

\footnotetext{
${ }^{3}$ We thank the final referee of our long paper [6] for drawing our attention to this.
} 
Lattice investigations of IR fixed points inside the conformal window $8 \lesssim N_{f} \leqslant 16$ all depend on naive scaling of Green's functions [22], so they correspond to scale-invariant vacua. A recent lattice study [23] of the running of $\alpha_{s}$ for two flavors with no naive scaling suggests that it freezes: the fixed point realises scale invariance in NG mode, i.e. with a scale-breaking vacuum. That is what $\chi \mathrm{PT}_{\sigma}$ assumes for three flavors.

The term "dilaton" often refers to a spin-0 particle or resonance which couples to $\theta_{\mu v}$ and acquires its mass "spontaneously" due to self interactions. Originally, this idea concerned a scalar component of gravity [24], but now it is a key ingredient of dynamical electroweak symmetry breaking (pp. 198 and 1622-3, PDG tables [9]). This approximates theories with scale-invariant vacua, as is evident in walking technicolor. Therefore it has nothing to do with our dilaton [25].

It is well known that a resonance cannot be represented by a local interpolating operator, so is the fact that $\sigma / f_{0}(500)$ has a finite width a problem for $\chi \mathrm{PT}_{\sigma}$ ? The answer is "no" because $\chi \mathrm{PT}_{\sigma}$ is an expansion in powers and logarithms of $m_{\pi, K, \eta, \sigma}$ with coefficients determined in the exact chiral-scale limit (1.2) where $\sigma$ has zero width [6]. In any perturbation theory, decay rates are calculated that way.

A related remark concerns what is current best practice for scenario 1 . The resonance $f_{0}(500)$ is treated as a member of the non-NG sector with an accidentally small mass. It causes $\chi \mathrm{PT}_{3}$ to produce divergent expansions for amplitudes involving $f_{0}(500)$ poles: the radius of convergence is too small. Instead, these amplitudes are approximated dispersively via contributions from the dominant $f_{0}(500)$ poles with corrections from nearby thresholds, subject to exact chiral $S U(3) \times$ $S U$ (3) constraints such as Adler zeros. One would certainly not use local fields in this framework.

However $\chi \mathrm{PT}_{\sigma}$ is a more ambitious theory. Having promoted $\sigma / f_{0}$ to the NG sector, we expect convergent asymptotic expansions for all mesonic amplitudes (scenario 2). The NLO corrections are still being worked out, but a first guess is to set all multi-dilaton vertices to zero. That is equivalent to adding the simplest dilaton diagrams to all $\chi \mathrm{PT}_{3}$ diagrams. It seems to produce amplitudes very similar to those of the dispersive approximations of scenario 1 .

\section{Acknowledgements}

We thank Claude Bernard, Gilberto Colangelo, Gerhard Ecker, Maarten Golterman, Martin Hoferichter, Heiri Leutwyler, and Daniel Phillips for useful comments about $\chi \mathrm{PT}_{\sigma}$ and the work we presented at CD2015. We also thank Nicolas Garron for informative discussions regarding RBC/UKQCD's analyses of $K \rightarrow \pi \pi$. LCT is supported by the Swiss National Science Foundation.

\section{References}

[1] S. L. Adler, J. C. Collins and A. Duncan, Energy-Momentum-Tensor Trace Anomaly in Spin 1/2 Quantum Electrodynamics, Phys. Rev. D 15, 1712 (1977).

[2] P. Minkowski, On the Anomalous Divergence of the Dilatation Current in Gauge Theories, Berne PRINT-76-0813, September 1976.

[3] N. K. Nielsen, The Energy Momentum Tensor in a Nonabelian Quark Gluon Theory, Nucl. Phys. B120, 212 (1977). 
[4] J. C. Collins, A. Duncan and S. D. Joglekar, Trace and Dilatation Anomalies in Gauge Theories, Phys. Rev. D 16, 438 (1977).

[5] R. J. Crewther and L. C. Tunstall, Origin of $\Delta I=1 / 2$ Rule for Kaon Decays: QCD Infrared Fixed Point, arXiv:1203.1321.

[6] R. J. Crewther and L. C. Tunstall, $\Delta I=1 / 2$ rule for kaon decays derived from QCD infrared fixed point, Phys. Rev. D 91, 034016 (2015) [arXiv: 1312.3319].

[7] I. Caprini, G. Colangelo and H. Leutwyler, Mass and width of the lowest resonance in QCD, Phys. Rev. Lett. 96, 132001 (2006) [arXiv: hep-ph/0512364].

[8] R. García-Martín, R. Kamiński, J. R. Peláez and J. R. de Elvira, Precise determination of the $f_{0}(600)$ and $f_{0}(980)$ pole parameters from a dispersive data analysis, Phys. Rev. Lett. 107, 072001 (2011) [arXiv:1107.1635].

[9] K. A. Olive et al. [Particle Data Group Collaboration], Review of Particle Physics, Chin. Phys. C 38, 090001 (2014).

[10] M. Gell-Mann, Symmetries of Baryons and Mesons, Phys. Rev. 125, 1067 (1962), footnote 38.

[11] A. Calle Cordon and E. Ruiz Arriola, Scalar meson mass from renormalized One Boson Exchange Potential, AIP Conf. Proc. 1030, 334 (2008) [arXiv: 0804 .2350].

[12] A. Calle Cordon and E. Ruiz Arriola, Renormalization vs Strong Form Factors for One Boson Exchange Potentials, Phys. Rev. C 81, 044002 (2010) [arXiv : 0905 . 4933].

[13] R. J. Crewther, Chiral Reduction of $K \rightarrow 2 \pi$ Amplitudes, Nucl. Phys. B 264, 277 (1986).

[14] J. F. Donoghue and H. Leutwyler, Energy and momentum in chiral theories, Z. Phys. C 52, 343 (1991).

[15] C. G. Callan, Jr., S. R. Coleman and R. Jackiw, A new improved energy-momentum tensor, Annals Phys. 59, 42 (1970).

[16] M. Hoferichter, D. R. Phillips and C. Schat, Roy-Steiner equations for $\gamma \gamma \rightarrow \pi \pi$, Eur. Phys. J. C 71, 1743 (2011) [arXiv:1106.4147].

[17] J. A. Cronin, Phenomenological Model of Strong and Weak Interactions in Chiral $U(3) \times U(3)$, Phys. Rev. 161, 1483 (1967).

[18] M. K. Gaillard and B. W. Lee, $\Delta I=1 / 2$ Rule for Nonleptonic Decays in Asymptotically Free Field Theories, Phys. Rev. Lett. 33, 108 (1974).

[19] C. Bernard, T. Draper, A. Soni, H. D. Politzer and M. B. Wise, Application of chiral perturbation theory to $K \rightarrow 2 \pi$ decays, Phys. Rev. D 32, 2343 (1985).

[20] P. A. Boyle et al. [RBC/UKQCD Collaboration], Emerging understanding of the $\Delta I=1 / 2$ Rule from Lattice QCD, Phys. Rev. Lett. 110, 152001 (2013) [arXiv: 1212 . 1474 ].

[21] Z. Bai et al. [RBC/UKQCD Collaboration], Standard-model prediction for direct CP violation in $K \rightarrow \pi \pi$ decay, arXiv:1505.07863.

[22] L. Del Debbio, The conformal window on the lattice, Proc. Sci. LATTICE2010 (2010) 004 [arXiv:1102.4066].

[23] R. Horsley, H. Perlt, P. E. L. Rakow, G. Schierholz and A. Schiller, The SU (3) beta function from numerical stochastic perturbation theory, Phys. Lett. B 728, 1 (2014) [arXiv: 1309.4311].

[24] Y. Fujii, Dilaton and Possible Non-Newtonian Gravity, Nat. Phys. Sci. 234, 5 (1971).

[25] P. Carruthers, Broken scale invariance in particle physics, Phys. Rep. C 1, 1 (1971). 